\title{
Las smart cities, la comunicación y el diseño: un reto de la sociedad del conocimiento
}

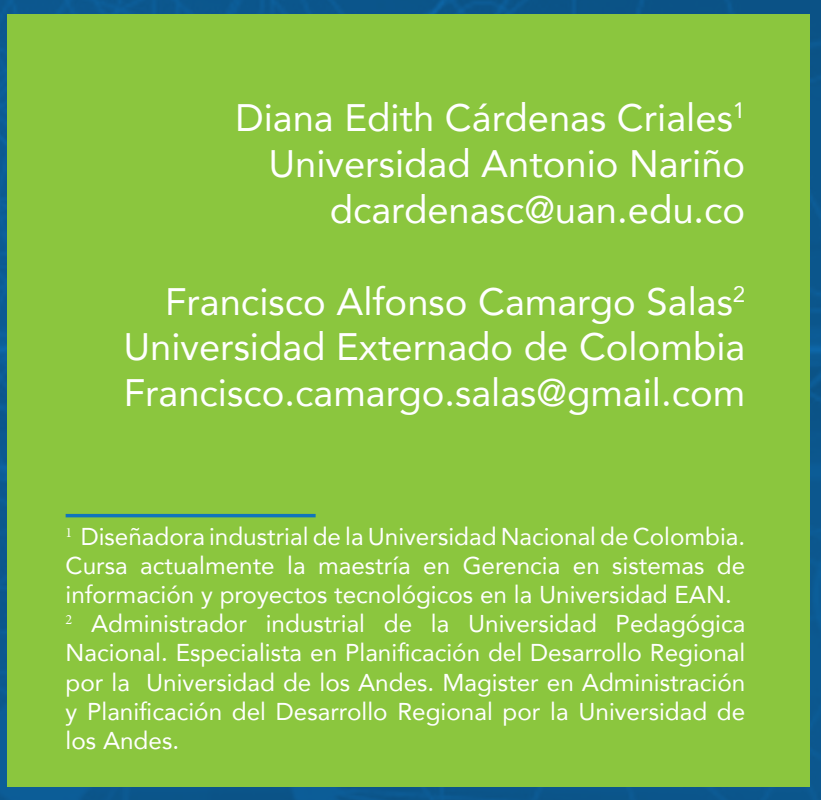

Cómo citar este artículo: Cárdenas, D., Camargo, F. A. (2016). Las smart cities, la comunicación y el diseño: un reto de la sociedad del conocimiento. Revista Virtualmente, 4(2), 42-55. 


\section{RESUMEN}

Entendiendo que los smart places o territorios inteligentes son aquellos espacios innovadoras capaces de encontrar un equilibrio entre los aspectos de competitividad económica, cohesión y desarrollo social y sostenibilidad ambiental, este artículo se propone evidenciar la comunicación como eje fundamental transformador, que debe ser considerada un proceso complejo articulador de todas las acciones humanas dentro de las smart cities. En este contexto, las disciplinas que tienen como objeto de estudio la creación simbólica, tangible e intangible de los seres humanos son de vital importancia, ya que inciden en todas las acciones humanas evidenciadas en su diversa producción, tales como la tecnología, la arquitectura, el diseño de productos, la multimedia, la moda y el arte.

Palabras clave: smart places, smart cities, comunicación, sociedad, diseño, ininovación. 


\section{Smart cities, communication and design: a challenge from the society of knowledge}

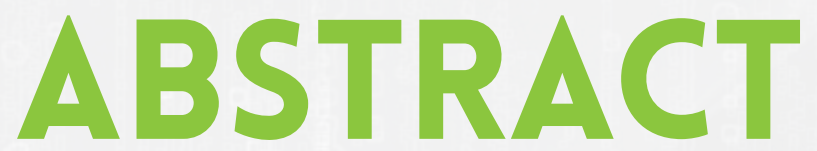

Understanding that smart places or intelligent territories are those innovative spaces capable of finding a balance between the aspects of economic competitiveness, cohesion and social development, and environmental sustainability, this article intends to highlight the communication as a fundamental transformative axis, which should be considered a complex process that articulates all human actions within the smart cities. In this context, the disciplines that have the symbolic, tangible and intangible creation of human beings as object of study are of vital importance, since they affect all human actions evidenced in their diverse production, such as technology, architecture, product design, multimedia, fashion and art.

Keywords: smart places, smart cities, communication, society, design, innovation. 


\section{Territoires intelligents, communication et conception: défis pour la société de la connaissance}

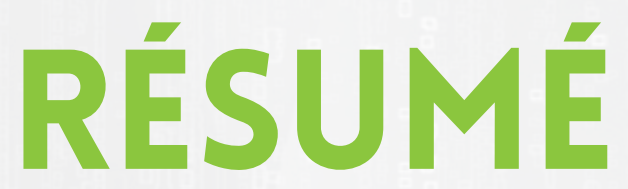

Cet article propose de mettre en évidence la communication comme axe fondamental de transformation dans le processus articulateur complexe des actions humaines. Le concept de «territoires intelligents » inclut des espaces innovants capables de trouver un équilibre entre les aspects de compétitivité économique, de cohésion, développement social et de respect de l'environnement.

Dans ce contexte, les disciplines ayant pour objet d'étude la création symbolique tangible et intangible des êtres humains sont d'une importance vitale dans la mesure où elles influent sur toutes les actions humaines mises en évidence au travers de productions technologiques, architecturales, de conception de produits, du multimédia, de la mode et de l'art.

Mots clefs: territoires intelligents, communication, société, création, innovation. 


\section{As cidades inteligentes, a comunicação e o design: um desafio da sociedade do conhecimento}

\section{RESUMO}

Considerando que os lugares inteligentes ou territórios inteligentes são aqueles espaços inovadores capazes de encontrar equilíbrio entre os aspectos de competitividade económica, coesão e desenvolvimento social e sustentabilidade ambiental, este trabalho tem como objetivo mostrar a comunicação como o eixo transformador que deve ser considerado um processo complexo articulador de todas as ações humanas dentro das cidades inteligentes. Neste contexto, as disciplinas que tem como objetivo de estudo a criação simbólica, tangível e intangível dos seres humanos são de importância vital, já que afetam todas as ações humanas evidentes em sua produção diversificada, como a tecnologia, arquitetura, design de produto, multimídia, moda e arte.

Palavras-chave: lugares inteligentes, cidades inteligentes, comunicação, sociedade, design, ininovación. 


\section{Introducción}

La paternidad de la mención de la llamada sociedad de la información se atribuye a los trabajos realizados durante la década de 1960 tanto por el estadounidense Daniel Bell como por el francés Alain Touraine, aunque también es cierto que decidieron utilizar la denominación sociedad posindustrial. Joyanes (1997) sintetizó su trabajo en el término ciberciudad, noción que ha recibido el impulso de una serie de informes para su concreción, entre estos, La informatización de la sociedad (Nora y Minc, 1980), Microelectrónica y sociedad para bien o para mal (Friedrichs y Schaff, 1982), Al Gore sobre la infraestructura de la información en los Estados Unidos de 1993 y Europa y la sociedad global de la información (Bangemann, 1994). Recomendaciones al consejo de Europa" de 1994. Su importancia es tal que la ONU ha organizado 2 cumbres mundiales de la sociedad de la información en ginebra 2003 y en Túnez 2005 para analizar su problemática (Cabero y Alonso, 2007).

Según Tubella i Casadevall y Vilaseca i Requena (2005), es una redundancia señalar que la base tecnológica de lo que hemos llamado sociedad de la información y el conocimiento está formada por las tecnologías de la información y de la comunicación (TIC), aclarando que sin un conocimiento de posibilidades de las TIC más allá de un conocimiento técnico y especializado de estas es prácticamente imposible gestionar ninguna actividad organizada dentro de las organizaciones.
El mundo actual es un mundo interconectado a través de la tecnología, donde la inmediatez valida la información recolectada. Una ciudad a la que se puede atribuir el adjetivo inteligente es aquella que dimensiona que el manejo de las TIC colabora en gran medida a fin de que los aspectos más importantes para su desarrollo comiencen a equilibrarse, teniendo en cuenta su crecimiento acelerado, donde el Gobierno implementa programas que interconecten a sus ciudadanos con actividades encaminadas a la objetivos comunes de la ciudad y de todos sus habitantes y visitantes.

Alrededor del mundo son pocas las ciudades a las cuales se les puede considerar realmente smart cities, que se encuentran en países que han desarrollado su economía a través de la investigación y la generación de conocimiento, además de volver accesible a sus habitantes el conocimiento creado. Si las nuevas tecnologías no son accesibles, incrementan la desigualdad porque son imprescindibles para el desarrollo económico. Esta brecha digital expresa una divisoria entre empresas, ciudades y personas en función de su posibilidad de integrarse en las nuevas premisas del desarrollo que dependen de la tecnología de la información. Una brecha que se mide por las diferencias en la calidad de la educación científica, en la investigación en universidades y empresas y en la disponibilidad de las más modernas infraestructuras (Vegara, 2009). 
Esta brecha digital se evidencia en el número de ciudades inteligentes alrededor del mundo y su ubicación geográfica. América del Sur, al sufrir de problemas de pobreza y de desigualdad en el acceso a la educación y a la tecnología, es fiel reflejo de que es necesario generar iniciativas para disminuir estas problemáticas y luego poder generar otras para que sus ciudades se conviertan en ciudades inteligentes (figura 1).

Figura 1. Smart cities en el mundo.

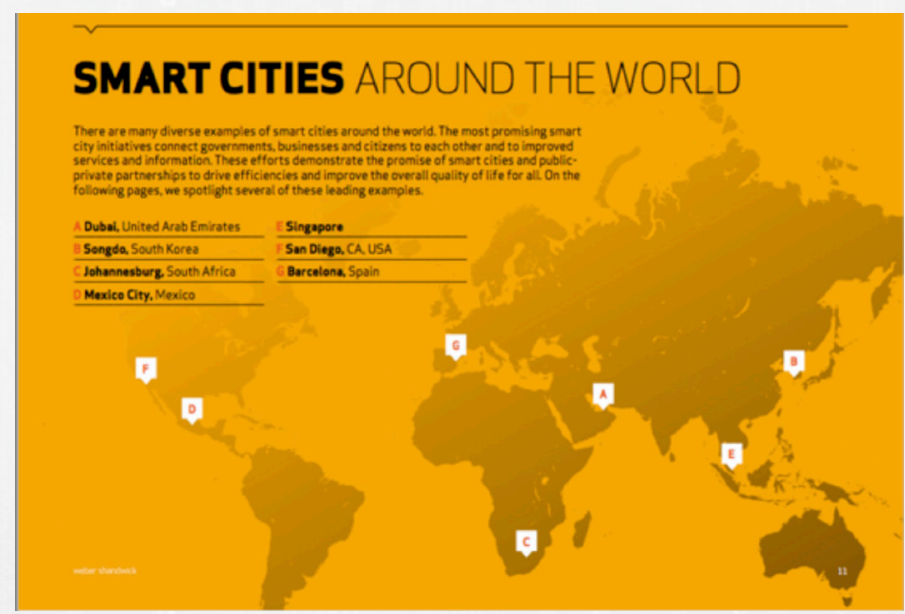

Fuente: Weber Shandwik (2015)

Se denominan smart places o territorios inteligentes a aquellos que están enfocando con coherencia los retos de la globalización y los riesgos que genera. Se trata de ciudades innovadoras capaces de encontrar un equilibrio entre los aspectos de competitividad económica, cohesión, desarrollo social y sostenibilidad ambiental (Vegara, 2009).
Son ciudades que basan su desarrollo urbano en la sostenibilidad teniendo en cuenta las necesidades de organizaciones, empresas y los mismos habitantes tanto en el aspecto económico como en los aspectos operativos sociales y ambientales de una forma eficiente. Puede ser calificada como inteligente en la medida en que sus inversiones crecen en el capital humano, en la educación en todos los niveles en aspectos sociales, en estructuras de energía como electricidad y gas, en tecnologías de comunicación electrónica e internet e infraestructura de transportes que promuevan y contengan una calidad de vida elevada y un desarrollo económico ambiental durable y sostenible en una gobernanza participativa, en una gestión prudente y reflexiva de los recursos naturales y en un buen aprovechamiento del tiempo de los ciudadanos (sustentAR tv, 2014) (figura 2).

Figura 2. Componentes de una smart city.

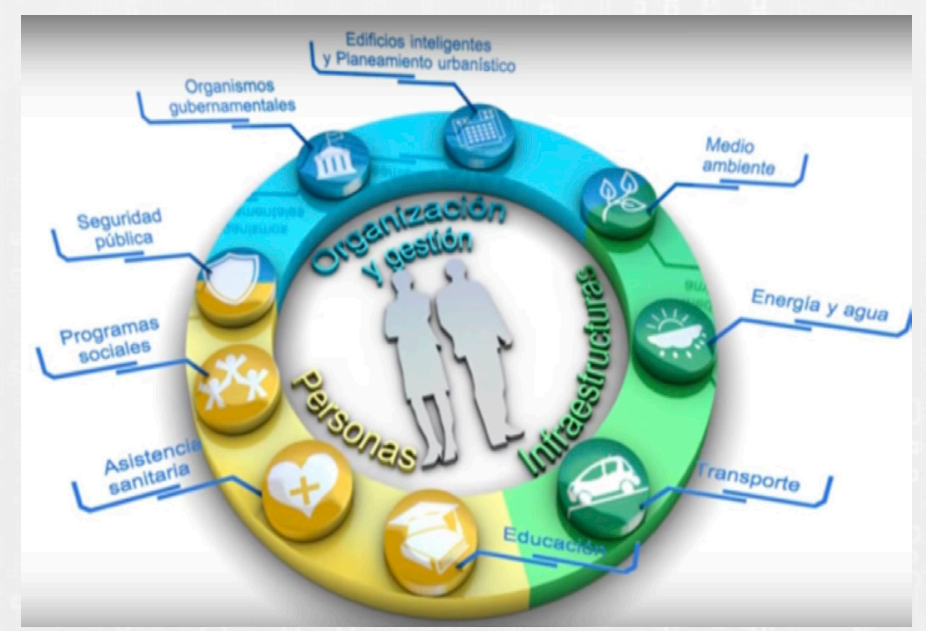

Fuente: Endesa Educa (2014) 
Desde esta perspectiva, las smart cities son aquellas que han logrado articular y permitir el acceso a mejores servicios tanto a los ciudadanos que las habitan, trabajan y se recrean dentro de estas como a sus visitantes, mejorando el nivel de vida gracias a que generan diferentes alternativas basadas en la sostenibilidad para realizar sus actividades cotidianas. Estas alternativas deberían proponerse como políticas gubernamentales en consecuencia del crecimiento desmesurado de las ciudades.

En 1950, aproximadamente el 30\% de la población podía considerarse urbana. En el 2000 el porcentaje de población urbana era ya del $50 \%$, para el año 2025 , cerca del $70 \%$ de la población mundial será urbana alojadas en por lo menos 30 mega ciudades con más de 8 millones de habitantes, y más de 500 ciudades con más de un millón de habitantes en el mundo. Un proceso de tales proporciones constituye un desafío social, infraestructural y ambiental extraordinario (Vegara \& de las Rivas, 2009).

Al ser tan alto el número de personas conviviendo en espacios concentrados en las ciudades, es evidente que debe generarse estrategias de consumo responsable de la energía Suzuki (2015) afirma que actualmente al año se gastan casi $€ 300$ millones en energía malgastada, €150 millones en comida desperdiciada, €100 millones debido a la congestión de las ciudades, además de causar la muerte de 7 millones de personas por causa de la polución a causa de que los servicios prestados por la ciudad no están conectados unos con otros (figura 3).
Figura 3. Dinero perdido a causa de la mala gestión de las ciudades.

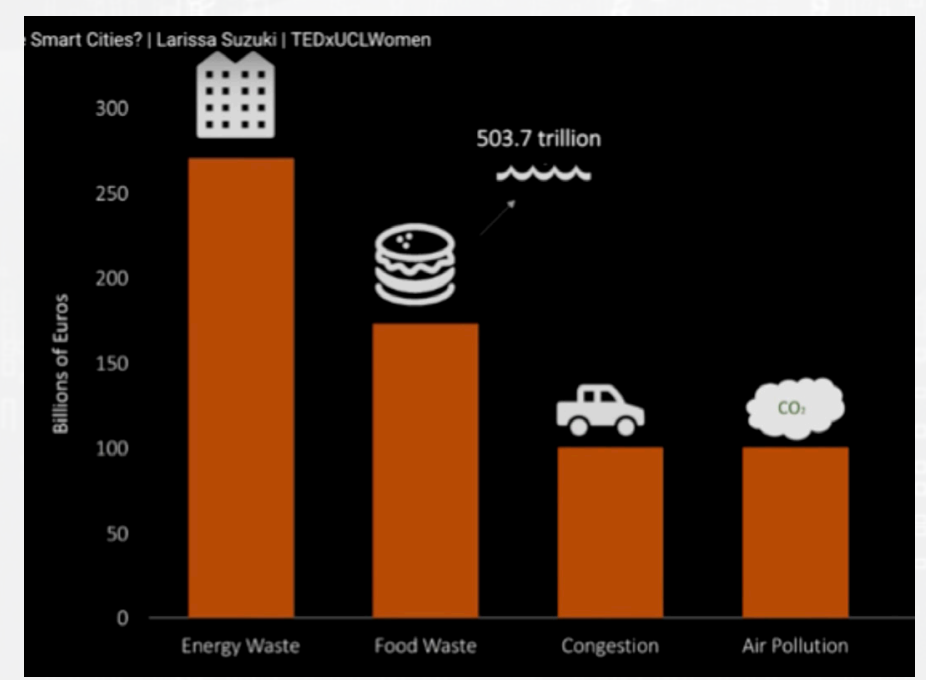

Fuente: Suzuki (2015).

Teniendo en cuenta lo expuesto, las organizaciones que abarcan los sectores públicos y privados, así como las entidades sin ánimo de lucro, desempeñan un papel protagónico para asegurar la capacidad que tienen las ciudades para manejar sus complejidades y permitir una alta calidad de vida de los ciudadanos que habitan en ellas. Las estimaciones indican que el potencial de mercado global para las ciudades inteligentes en el desarrollo de la infraestructura, la integración de la tecnología, la administración electrónica, la energía y los servicios de seguridad podría alcanzar los US\$3.3 billones de dólares para 2025. Gran parte de ese potencial se realizará por las inversiones de colaboración en soluciones e innovaciones que aborden la inclusión, la sostenibilidad y la capacidad de recuperación dentro de las ciudades (Weber Shandwik, 2015). 
El reto de las smart cities no solo depende de las organizaciones del sector público y privado, sino de la capacidad colectiva para identificar, implementar y ampliar este tipo de soluciones e inversiones dentro y entre ciudades. Ese trabajo requiere una mayor capacidad para identificar los socios adecuados, comunicar ideas complejas con y entre los interesados e involucrar a la ciudadanía mayor en las campañas para impulsar el apoyo y la acción en todos los niveles desde las comunidades locales a los ecosistemas en red de la ciudad que conectan a las personas a través de la geografía y la tecnología (Weber Shandwik, 2015).

Al entender que el principal reto de las smart cities es trabajar en equipo, se deben generar canales claros de intercambio de información entre ciudadanos, organizaciones y el Gobierno, con el objetivo de crear sentido y retroalimentación entre sí acerca de los cambios de hábitos y nuevas alternativas que deben ser generadas para mejorar los servicios de la ciudad. Visto desde este enfoque, un gran reto de las ciudades inteligentes es el de poder diseñar comunicación efectiva que conecte a todos sus actores.

Por este motivo, es necesario revisar el concepto de comunicación, que, a partir del primer tercio del siglo XX hasta la actualidad, se ha ido construyendo desde perspectivas muy diferentes. Desde la teoría físico-matemática, conocida como teoría matemática de la información, hasta la teoría psicológica basada en la percepción, pasando por una teoría social que relaciona lenguaje y comunicación, por el enfoque de la antropología cognitiva y los planteamientos fundamentados en la interacción. También han destacado las aportaciones en el campo de los efectos de la comunicación de masas, y las teorías críticas de la comunicación, promovidas desde la Escuela de Frankfurt (Rizo, 2012).
El enfoque de la comunicación más cercano al objetivo que se pretende alcanzar en las smart cities es el propuesto por la Escuela de Palo Alto, debido a que sus axiomas de comunicación rompen con la visión unidireccional o lineal de la comunicación. La comprensión de la comunicación se resume en considerarla la base de la interacción social y, de este modo, fundamento para la construcción del mundo social (Rizo, 2012).

La comunicación es acción y la acción es comunicación. Los hechos no lo son, sino que significan, y en esta medida podemos decir que comunican. Si la comunicación se inscribe en las ciencias de la acción, una y otra devienen indisociables y esta fuerza conjunta unificada abre una perspectiva más allá de la concepción reduccionista de la comunicación basada en el mass media como simple difusión de mensaje. He definido la unidad de la acción y la comunicación en dos facetas, distintas pero complementarias: la acción factual, de fuente de energía (los hechos, las realizaciones, los productos, las construcciones) y la acción comunicativa, de energía débil (los símbolos, los signos, las imágenes y el lenguaje). (Costa, 1995)

Más allá de comprender la comunicación como una herramienta para difundir mensajes, aliados de la publicidad masiva, ella es, de hecho, el nexo de unión entre las ciencias sociales con espíritu holístico, ahora beneficiado por la concepción sistemática, que no solo ve el todo, sino que identifica las partes y sus interrelaciones, y opera sobre ellas. Se afirma así que es la comunicación la herramienta privilegiada de la gestión (Costa, 2015).

Así es como se está configurando un nuevo mapa del mundo de las comunicaciones. Sus rasgos característicos son la emergencia de la diversidad y la integración democrática de los diferentes tipos de organizaciones que definen el sistema social, el cual se constituye necesariamente a través de la comunicación (Costa, 2015). 
Más allá de comprender la comunicación como una herramienta para difundir mensajes, aliados de la publicidad masiva, ella es, de hecho, el nexo de unión entre las ciencias sociales con espíritu holístico, ahora beneficiado por la concepción sistemática, que no solo ve el todo, sino que identifica las partes y sus interrelaciones, y opera sobre ellas. Se afirma así que es la comunicación la herramienta privilegiada de la gestión (Costa, 2015).

Así es como se está configurando un nuevo mapa del mundo de las comunicaciones. Sus rasgos característicos son la emergencia de la diversidad y la integración democrática de los diferentes tipos de organizaciones que definen el sistema social, el cual se constituye necesariamente a través de la comunicación (Costa, 2015).

\section{Desarrollo}

Para que las ciudades en la actualidad se transformen en smart cities, hay que reconocer la importancia de la comunicación dentro de este cambio, por lo cual debe ser entendida como un proceso complejo articulador de todas las acciones humanas de construcción social que pueden romper paradigmas de comportamiento. Con la comunicación efectiva entre los agentes de las smart cities (ciudadanos, empresas y Gobierno), se puede generar el cambio de mentalidad necesario para que el concepto de un mejor nivel de vida esté asociado a la sostenibilidad y el uso racional de la energía.
La cultura y el aprendizaje humano se realizan mediante la comunicación, o interacción simbólica, por la que cada ser humano adquiere el propio sentido del ser, su carácter e identidad. El self(Mead, 1934) o el "yo espejo" (Cooley, 1909) es la constitución de un yo a partir de la interacción con los demás. Para Mead (1934), cada uno de nosotros llega a ser consciente de una especie de otro generalizado, a saber, la sociedad en general (Rizo, 2012).

Por tanto, aquellas disciplinas que tienen como objeto de estudio la creación simbólica tangible e intangible de los seres humanos, la decodificación personalizada de estos símbolos, a partir de su aprendizaje consiente e inconsciente generado por las experiencias en contextos geográficos, políticos y religiosos determinados, son de vital importancia, ya que inciden en todas las acciones humanas, donde se toma posición en favor o en contra de los cambios dentro de la ciudad y se evidencian todas sus producciones, como la tecnología, la arquitectura, el diseño de productos, multimedia, la moda y el arte.

Retomando lo que se postula en la Escuela de Palo Alto todas, las acciones humanas comunican gracias a la carga simbólica que contienen. En este sentido, el diseño es una disciplina especializada en crear sentido de manera consiente y de comunicar de manera efectiva a los usuarios a través de diferentes medios de comunicación, por ejemplo objetos, lugares habitacionales y piezas multimediales, que, gracias a la tecnología actual, cada vez son más interactivas. El diseñador es capaz de resolver problemas humanos de forma estratégica, entendible, usable y adaptable a contextos específicos. Por este motivo, se retoma la importancia de la disciplina del diseño industrial dentro de los procesos de transformación de las ciudades actuales a smart cities. 
El diseño industrial es un proceso estratégico destinado a resolver problemas, aplicable a productos, sistemas, servicios y experiencias, cuyos resultados redundan en innovación, éxito en los negocios y en una mejor calidad de vida. Es una profesión transdisciplinar que involucra a la innovación, a la tecnología, a los negocios, a la investigación y a los clientes en el aprovechamiento de la creatividad y la visualización para resolver problemas de cara a plantear soluciones, reformulando problemas para convertirlos en nuevas oportunidades con la intención de hacer un mejor producto, sistema, servicio, experiencia 0 negocio y proporcionando siempre valor y/o una ventaja competitiva. En los resultados del diseño industrial están implícitos de forma intencionada los aspectos económicos, sociales, ambientales y éticos que han de ir siempre encaminados a crear un mundo mejor. (WDO, 2015).

Al reconocer que el reto de las smart cities no solo es de avances tecnológicos, es necesario trabajar de la mano de disciplinas como el diseño industrial que pone al ser humano como centro de todos los procesos. Al conocer al ser humano, conoce sus necesidades a fondo a través de la empatía. Por tanto, estas necesidades son resueltas con la aplicación de métodos de diseño donde el eje conductor siempre es el ser humano que responde a través de intervenciones tangibles e intangibles. El diseñador industrial se vuelve un actor estratégico dentro de los procesos de innovación, gracias a que es capaz de comprender los procesos tecnológicos, sociales y del entorno desde una perspectiva sistémica, en la que las relaciones constituyen la base de su acción.

Acorde con las metodologías del diseño industrial, Nigel Vaz (2014) sostiene que la parte más importante es la social y la experiencia para invitar a las personas a las ciudades. La mayoría de los proyectos fallan, ya que las ciudades no tienen en cuenta el real reto que es lidiar con 10 o 20 millones de personas y sus formas de pensar, la mayoría de los proyectos han sido construirlos y se espera a que las personas lo usen después; al no estudiar a conciencia a los habitantes de las ciudades, se dan cuenta de que dichos proyectos se construyeron sin ningún propósito y nadie visita esos espacios, por lo que lo mejor es pensar proyectos en áreas urbanas existentes (The Drum, 2014).

Así es como el diseñador industrial se convierte en un traductor de la información recolectada por observación del entorno, la decodifica en parámetros de diseño y la reconfigura nuevamente en respuestas efectivas basadas en la tecnología existente y disponible, con la característica de que toda respuesta puede ser entendida por la sociedad que fue estudiada. El objetivo primordial del diseñador dentro de las smart cities es humanizar la tecnología utilizada para conectar a los humanos entre sí y que esta sea lo más cercana posible a su entorno para que no le parezca extraña y la adopte con naturalidad.

Además, el diseñador se convierte en un actor importante en el proceso de ayudar a la comunidad a generar respuestas que le ayuden a solventar sus problemas con metodologías participativas aplicadas en la innovación social. Por su parte, entender temas como la competitividad económica, el equilibrio social, la calidad de vida y la sostenibilidad ambiental dependerán de nuestra capacidad para innovar y compartir estas innovaciones con otras ciudades en un mundo cada vez más global e interrelacionado (Vegara, 2009).

En The Drum (2014) también refuerza la postura de que el gran reto al hablar de las smart cities es un problema sobre personas y cómo se comunican entre sí. Se argumenta que 
pensar en smart cities es pensar en interconexión entre los ciudadanos a través de los dispositivos electrónicos que ya poseen, "porque las cities giran alrededor de las personas, no es sobre tecnología, es sobre motivación". Las iniciativas que se han implementado dejan claro que, al tomar el lado humano de la competitividad, los ciudadanos son más prestos a adoptar nuevos hábitos donde los resultados pueden ser comparados con otras personas habitantes de su mismo sector, además de exaltar que las iniciativas implementadas se desarrollan a partir del entendimiento de las acciones cotidianas del ser humano.

Comprendiendo que los servicios que se prestan en las ciudades son sustentados en el capital humano de las organizaciones, es necesario interconectar personas para asimismo conectar los servicios ofrecidos en la ciudades, con el objetivo de optimizar el gasto energético dentro de estas (Suzuki, 2015). Esta conexión se da gracias a la tecnología que surge del conocimiento de la ciudad misma, puesto que se parte del estudio de las necesidades que surgen a partir de la sociedad y se generan respuestas que ayuden a esta.

Así es como el movimiento de las ciudades inteligentes está ganando impulso, sobre todo, como una respuesta proactiva a los desafíos relacionados con el crecimiento demográfico, la urbanización y el cambio climático. Hay una oportunidad para que los líderes de todos los sectores aceleren el progreso. Gran parte de ese liderazgo requerirá asociación de las iniciativas inteligentes de la ciudad fuera de los ámbitos de la planificación urbana y la investigación académica. Las ciudades inteligentes deben llegar a ser tangibles, relevantes y accesibles (Weber Shandwik, 2015).
De esta manera, se demuestra que el reto de las smart cities no es solo un proceso cuantitativo, sino que las ciudades son los nodos que articulan y organizan la economía mundial, con un papel de liderazgo creciente, y sin el compromiso de las ciudades, los principios del desarrollo sostenible y la continuidad de la calidad de vida en el planeta son inviables. Por ello, organizar las ciudades del siglo XXI es una de las grandes cuestiones de la humanidad (Vegara, 2009).

\section{Conclusiones}

Aunque existe la corriente de que el problema de las smart cities es de tecnología y de inversión en infraestructura principalmente, no hay que olvidar que las ciudades son habitadas por personas que realizan acciones habitualmente. El estudio de estas acciones contribuye a la generación de iniciativas que pueden cambiar la conciencia sobre el gasto energético de las ciudades. Así, la implementación de proyectos con miras a transformar ciudades debe hacerse de manera gradual, por lo que el ser humano, al ser resistente a cambios drásticos, no adopta aquello que le es totalmente ajeno, lo que puede generar pérdidas en grandes inversiones en las ciudades. Como afirma Costa (2015), la tecnología ha excluido a la sociología en nuestra era digital. Es urgente recuperar las disciplinas que estudian al ser humano y sus interacciones sociales, ya que existe tecnología y economía gracias al ser humano, y no al contrario.

El gobierno tanto de los países como de las ciudades desempeña un papel protagónico dentro de la transformación de las ciudades, por lo que generar políticas de articulación de disciplinas e incentivos a la investigación conjunta entre 
la academia, la industria y la sociedad puede producir los cambios esperados dentro de las ciudades. Según Fernández y Bonsiepe (2008), el diseño industrial puede contribuir a solventar problemas de fondo en América Latina como lo son la pobreza y la desigualdad en la educación, de esta forma se contribuye al desarrollo de las smart cities en países en vías de desarrollo.

El fin último de la tecnología dentro de las smart cities es que la gente hable el mismo lenguaje, que las ciudades se hagan entender de la misma manera para los lugareños y visitantes, es generar comunicación efectiva, por lo que el diseño industrial se convierte en un articulador de conocimientos de diferentes disciplinas que traduce a un lenguaje entendible por todos, demuestra el poder de la acción colectiva para resolver problemas desde la inclusión, la sostenibilidad y la resiliencia y, sobre todo, unifica bajo un objetivo común.

\section{Referencias}

Bangemann, M. (1994). Europa y la sociedad global de la información: recomendaciones al Consejo Europeo. Bruselas: Parlamento Europeo.

Cabero Almenara, J. y Alonso García, C. M. (2007). Nuevas tecnologías aplicadas a la educación. Madrid: McGraw-Hill.

Costa, J. (1995). Comunicación corporativa y revolución de los servicios. Madrid: Ediciones Ciencias Sociales.

Costa, J. (2015). El paradigma DirCom: el nuevo mapa del mundo de la comunicación y el management estratégico global. Barcelona: Joan Costa Institute.
Elche Dinámico EFDEC (2014a). Documental smart cities Part 1 de Escarabajo Verde RTVE. Recuperado de: https://www.youtube. com/watch?v=S_RnkRkwZOM

Elche Dinámico EFDEC (2014b). Documental smart cities Part 2 de Escarabajo Verde RTVE. Recuperado de: https://www.youtube. $\mathrm{com} /$ watch?v=DPSTfVXGtew

Endesa Educa (2014). ¿Qué es una smart city? Recuperado de: https://www.youtube.com/watch?v=|Kpoi8lf_tl

Fernández, S., \& Bonsiepe, G. (2008). Historia del diseño en América Latina y el Caribe: industrialización y comunicación visual para la autonomía. São Paulo: Ed. Blücher.

Friedrichs, G. y Schaff, A. (1982). Microelectrónica y sociedad, para bien o para mal: informe al Club de Roma. Alhambra.

Gómez-Vela, M. y Sabeh, E. N. (2016). Calidad de vida: evolución del concepto y su influencia en la investigación y la práctica. Recuperado de https://campus.usal.es/ inico/investigacion/ invesinico/calidad.htm

Joyanes, L. (1997). Cibersociedad: los retos sociales ante un nuevo mundo digital. Madrid: McGraw-Hill.

Koon Hean, C. (2015). How we design and build a smart city and nation. En TEDxSingapore. Recuperado de: https://www. youtube.com/watch? $v=m 45 S$ shJqOP4

Nora, S. y Mine, A. (1980). La informatización de la sociedad. Madrid: Fondo de Cultura Económica.

Observatorio Tecnológico de la Energía (18 abril 2012). Mapa tecnológico "ciudades inteligentes". Madrid: Ministerio de Industria, Turismo y Comercio. Recuperado de: http://www. idae.es/uploads/documentos/documentos_Borrador_Smart Cities_18_Abril_2012_b97f8b15.pdf 
Rizo, M. (2012). El interaccionismo simbólico y la Escuela de Palo Alto: hacia un nuevo concepto de comunicación. En Portal de la Comunicación. Recuperado de: http://portalcomunicacao. com/uploads/pdf/17_esp.pdf

SENA (Servicio Nacional de Aprendizaje) (2016). Elementos de un sistema de información. Recuperado de: https://vimeo. com/6794622

SustentAR tv. (2014). Ciudad inteligente, ¿qué son? Recuperado de: https://www.youtube.com/watch?v=WBOE7CTYg4k

Suzuki, L. (2015). What are smart cities? En TEDxUCLWomen. Recuperado de: https://www.youtube.com/watch?v=Kqkoghq$0 G 4 \mathrm{~A}$

The Drum (2014). Cities are getting smarter: The day before tomorrow. Recuperado de: https://www.youtube.com/watch?v=HgPx TuF-Js

Tubella i Casadevall, I. y Vilaseca i Requena, J. (coords.) (2005). Sociedad del conocimiento: cómo cambia el mundo ante nuestros ojos. Barcelona: UOC.
Vaz, N. (2014). Cities Are Getting Smarter I The Day Before Tomorrow. Recuperado de: https://www.youtube.com/watch?v=HgPx TuF-Js

Vegara, A. (2009). Territorios inteligentes. Ambienta, 89, 34-60.

Venkatraman, V. (2014). Mobility beyond transport in smart cities. En TEDxCopenhagenSalon. Recuperado de: https://www. youtube $. \mathrm{com} /$ watch $? \mathrm{v}=\mathrm{z} 5 \mathrm{O} 4 \mathrm{Y} \mid 6 \mathrm{ZB} 4 \mathrm{k} \&$ spfreload $=1$

Weber Shandwik (2015). Innovation trends report: Networked smart cities. Recuperado de: http://www.webershandwick.com/ uploads/news. f.iles/WS-Networked-Smart-Cities-Report.pdf

WDO. (2015). Definition of Industrial Design. Recuperado de: http:// wdo.org/about/definition/ 